\title{
A hybrid beam design for slow positron transport
}

\author{
F J Mulligant and $M \mathbf{S}$ Lubell \\ † Department of Experimental Physics, St Patrick's College, Maynooth, Co. Kildare, \\ Ireland \\ ¥Department of Physics, City College of CUNY, New York, NY 10031, USA
}

Received 10 August 1992, accepted for publication 22 October 1992

\begin{abstract}
We report on the design of a hybrid transport system for slow positrons based upon the high-intensity magnetically transported positron beam, which existed at the Brookhaven (BNL) high-flux beam reactor (HFBR) in 1986. The resulting modified transport, incorporating an initial electrostatic stage followed by a magnetic stage and completed by brightness enhancement moderation leading to a final electrostatic section, represented the minimum alteration of the existing beam to enable it to be used for crossed-beams differential scattering studies involving a variety of atomic and molecular systems, including atomic hydrogen. While subsequent developments at BNL obviated the need for this approach, a recent proposal for constructing a very high-intensity positron beam at the Paul Scherrer Institute (PSI), based upon magnetic confinement premoderation, has rekindled interest in the conclusions of our study.
\end{abstract}

\section{Introduction}

Since the early 1970s considerable effort has been devoted to the development of monochromatic, lowenergy positron beams [1-16] for applications in condensed matter physics $[13,15]$ and atomic physics [17-19]-in the latter case often serving purposes that complement similar applications of electron beams $[17$, $19,20]$, either polarized or unpolarized. Whether incorporating moderation techniques $[9,12,21,22]$ or not [19], the low-energy positron beams have generally achieved their monochromaticity through the use of magnetic fields to reject the unwanted high-energy contamination $[11,12,21,23,24]$. Moreover, as Canter [25] has pointed out, even experimental approaches which do not incorporate truly monochromatic beams, but which rely rather on the use of time-of-flight (TOF) techniques $[17,26,27]$ to discriminate against highenergy positrons, have still almost always employed magnetic guide fields for transporting and confining the beam. While ToF experiments have achieved remarkable success, it is probable that future advances in positronatom scattering, particularly those involved with the measurement of low-energy differential cross sections, will increasingly utilize interaction environments that are virtually free of magnetic fields. Indeed the first experimental studies carried out under such conditions have been reported within the last few years [28, 29].

As part of our goal to develop a positron-atom scattering experiment capable of achieving the precision normally associated with electron-atom scattering, we were led several years ago to consider the Brookhaven high-flux beam reactor (HFBR) intense, low-energy positron beam, which is produced through moderation techniques. In its original configuration, the extraction optics of the Brookhaven (BNL) beam and even the moderated source itself resided in a magnetic environment. The emittance requirements of a low-energy crossed-beams geometry scattering experiment, however, dictated that the final transport stage be electrostatic in nature. We directed our efforts, therefore, towards adapting the beam to our needs, since its intensity and energy resolution were well suited to our future objectives. As we will demonstrate it was necessary to couple the magnetic transport to an initial electrostatic section in addition to the final electrostatic stage. Although the calculations are specific to our design requirements, the conclusions that we draw about the constraints on such a hybrid electrostatic-magnetostatic-electrostatic-(EBE)

transport system have more general applicability. In fact, although developments at BNL precluded the installation of a hybrid system $\dagger$, we are motivated to publish the results of our design study by a proposal to construct a very high-intensity positron beam at the Paul Scherrer Institute (PSI) based upon magnetic confinement premoderation [30].

Hughes et al [31] have shown that the emittance $\varepsilon$ of a beam in a magnetic field must be generalized to

† Shortly after our design had been completed it became necessary to transfer the entire positron experiment to a new building at BNL. The new beam was intended to serve for at least three separate experiments. Electrostatic rather than magnetic deflectors were chosen to select the experiment which received the beam at any time. This choice, together with the scale and complexity of the project led to a decision to use a complete electrostatic transport system. Consequently the design described above was not implemented. 
inciude the effects of the magnetic vector potential which appears in the expression for the canonical angular momentum. If, for example, a cathode is embedded in a magnetic field of strength $B_{0}$, the generalized emittance, $\varepsilon^{*}$ of the resultant beam in a field-free region then assumes the approximate form [31].

$$
\varepsilon^{*}=\rho_{0} \sqrt{E_{0} / E}+\frac{1}{2}(e / m)\left(\rho_{0}^{2} B_{0} / v\right)
$$

where $\rho_{0}$ is the radius of the electron beam at the cathode, $E_{0}$ is its characteristic energy at the cathode, $E$ its energy in the field-free region, $v$ its velocity in that region, and $-e$ and $m$ are respectively the charge and mass of the electron. The first term in equation (1) is the usual non-relativistic, non-invariant emittance $\varepsilon$, while the second term is the field-dependent piece, which may contribute substantially even for modest values of $B_{0}$, provided $v$ is small enough and $\rho_{0}$ is large enough. For many practical cases equation (1) thus demonstrates that the cathode itself must reside in a region essentially free of magnetic fields, if the ultimate application of the beam is at low energy and in an interaction region free of magnetic fields. If a magnetostatic section is to be employed for purposes of long-distance transport, the beam line must then assume a hybrid EBE configuration.

For the purpose of design modifications that would meet the needs of crossed-beams atomic physics [32], we established the following positron requirements: $10^{7}$ $\mathrm{e}^{+} \mathrm{s}^{-1}$ within a radius $\rho$ of $3 \mathrm{~mm}$ and a divergence (beam-plus-pencil half angle), $\alpha$ of $2^{\circ}$ at an energy of $10 \mathrm{eV}$, corresponding to a non-invariant emittance $\varepsilon=$ $\rho x$ of approximately $10 \mathrm{mrad} \mathrm{cm}$ at $10 \mathrm{eV}$ or equivalently to an invariant emittance $\varepsilon^{\prime}$ of $33 \mathrm{mrad} \mathrm{cm} \mathrm{eV} V^{1 / 2}$. We further required that the energy of the beam be variable between 1 and $500 \mathrm{eV}$ and that the crossed-beams scattering region be free of magnetic fields at the level of $100 \mathrm{mG}$. The magnetic field criterion for the scattering region dictates the use of electrostatic beam optics for the final stage of the transport system.

\section{Design considerations}

As developed by a consortium of physicists from AT\&T Bell Laboratories, BNL, Brandeis University and the City College of CUNY, the Brookhaven beam in 1986 operated at an intensity of $10^{8} \mathrm{e}^{+} \mathrm{s}^{-1}$ and at $400 \mathrm{eV}$ produced a beam spot $1.0 \mathrm{~cm}$ in diameter with a divergence half angle of $2^{\circ}$. Its intrinsic energy resolution of $\sim 75 \mathrm{meV}$ was more that adequate for most positron-atom collision studies. By contrast, its invariant emittance $\varepsilon^{\prime}=\varepsilon \sqrt{E}$ of $350 \mathrm{mrad} \mathrm{cm} \mathrm{eV} \mathrm{eV}^{1 / 2}$ was about an order of magnitude greater than that demanded by typical crossed-beams scattering experiments. Moreover, with the source of positrons residing in a magnetic feld of $20-50 \mathrm{G}$, the consequent inflation of its generalized emittance $\varepsilon^{*}$, made the beam totally inappropriate for such experiments in its original configuration. A substantial design modification was clearly required.

The relatively poor emittance of the existing beam strongly suggested the use of "brightness enhancement remoderation' $[6,33-37]$ to lower the value of $\varepsilon^{\prime}$ substantially. Originally proposed by Mills [33], brightness enhancement remoderation is a non-conservative process in which the positron beam is first accelerated to reduce its non-invariant emittance $\varepsilon$ and then allowed to impinge on a remoderator where the positions are thermalized in the bulk of the solid. A fraction of the positrons reach the surface and escape with an energy of 1 to $2 \mathrm{eV}$, characteristic of the negative affinity of the surface for positrons. There are now fewer positrons in the beam $[33,36,37]$ but the invariant emittance $\varepsilon^{\prime}$ is substantially lower.

Consistent with equation (1) and the definition of the invariant emittance, $\varepsilon^{\prime}$ can be expressed as

$$
\varepsilon^{\prime}=\rho_{0}\left(E_{0} / E\right)^{\frac{1}{2}} \sqrt{E}=\rho_{0} \sqrt{E_{0}}
$$

for a spot size of radius $\rho_{0}$ on the moderator and a transverse escape energy $E_{0}$. The acceptance criterion established for the scattering experiment then translates into a requirement of $\rho_{0}=0.75 \mathrm{~mm}$ for a maximum value of $E_{0} \approx 0.2 \mathrm{eV}$. Should such a radial dimension prove to be impracticable, several stages of remoderation in principle could be used to achieve the desired value of $\varepsilon^{\prime}$. However, since each added stage introduces added complexity and, moreover, since each stage attenuates the beam by at least $70 \%[33,34,36]$, we carried out our design study with the objective of using a single stage of brightness enhancement based upon thin film transmission remoderation [38].

\section{Experimental design}

For the purposes of our study we assumed that the source and extraction of the BNL beam would be altered to accommodate magnetically field-free operation; this was consistent with modifications which were being made to the source region to take advantage of the highefficiency solid neon moderator which had just been developed by Mills and Gullikson [9]. However, we also assumed that the magnetic transport system would be maintained causing a minimum disruption of the existing beam line. Our task thus became the design of a hybrid beam line, starting with an electrostatic $(E)$ extraction system, followed by a magnetostatic (B) transport section, and culminating in an electrostatic $(E)$ geometry compatible with brightness enhancement remoderation. We carried out our design study with the aid of the SLAC electron (positron) ray-tracing code developed by Hermannsfeldt [39].

As suggested by equation (1), the magnetic portion of an EBE hybrid system should not affect the final phase space of the beam, a conclusion that also follows by the direct application of Busch's theorem [40]. The theorem states that an electron or positron travelling from a point $A$ to a point $B$ in an axial magnetic field, where

$\$$ This value is typical for emission angles of $\sim 15^{\circ}$ with respect to the normal surface of a W(110) moderator, as shown by Mills [33] 
the magnetic flux passing through two coaxial circles drawn through points $A$ and $B$ may be different, undergoes a change of angular momentum that is proportional to that difference in the magnetic flux. Thus, in contrast to the situation where a beam originating in a magnetic field is ultimately used in a region free of magnetic fields, the interjection of a magnetic transport line between two magnetically field-free regions does not change the magnetic flux between the initial and final positions of the beam. Hence such a magnetic transport system does not alter the angular momentum or invariant emittance of the beam.

For the electrostatic extraction from the source moderator as well as from the downstream remoderator, we adapted the modified Soa gun geometry described by Canter et al [41]. We faced two choices for making the transition to the magnetic field-either to use a gradual transition, as would be the case with an air-core magnetic coil, or to use a rapid transition, as would be the case with iron termination plates. The former approach was easier to model and in fact was suggested to us by a number of people on the grounds that a slow transition into a magnetic field would produce less aberration of the beam. Contrary to this argument, we found that a gradual transition distorted the beam substantially more than did a rapid transition. Moreover, with the use of termination plates we were able to place the remoderator close to the exit point of the magnetic field, thereby simplifying the design substantially. Figure 1 illustrates schematically our resulting design approach.

We chose $10 \mathrm{~mm}$ thick soft iron plates as field

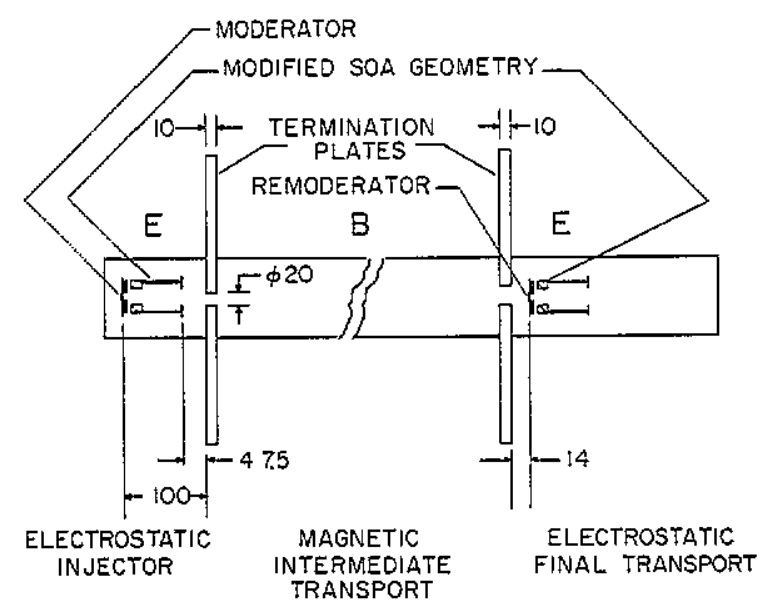

Figure 1. Schematic lay-out of the hybrid beam line, showing the electrostatic (E) modified Soa gun injection region, the magnetic $(B)$ intermediate transport section, and the electrostatic (E) final transport section including the 'brightness enhancement' remoderation stage. All dimensions are given in millimetres. It should be noted that the length of the magnetic section depends upon the beam energy (cathode voltage $V_{c}$ ) and guide field-strength chosen and, as described in the text, may be incremented in units of the helical period corresponding to those choices.
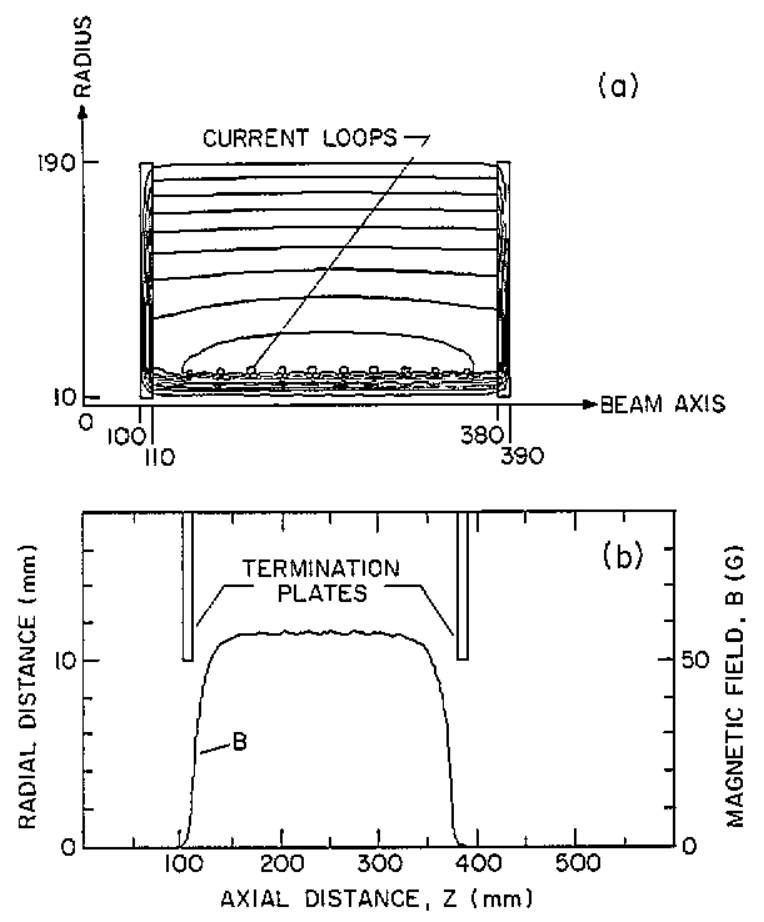

Figure 2. (a) Model of magnetic field with iron termination plates, showing field lines as calculated by the PoIsson code based upon ten energizing current loops. The dimensions for the model are given in millimetres. $(b)$ Axial magnetic field plot for the terminated field shown in (a).

terminators and used the computer code Porsson§ to model the magnetic field. The choice of plate dimensions is dictated by the requirement that the magnetic field must not be allowed to saturate the iron, which would then lead to leakage of field lines, thus defeating the purpose of the terminating plates. The results of the modelling appear in figures $2(a)$ and $(b)$, the former illustrating pictorially the field lines and the latter displaying quantitatively the behaviour of the field along the magnetic field. These results were obtained from a vector potential array which is the basic output of the POIsson code and which, when properly formatted in fact served as direct input to the SLAC/Hermannsfeldt ray-tracing program.

In order to simplify the design analysis, we used the tabulations of Canter et al [25] for the extreme rays that correspond to different tuning conditions of the modified Soa gun, always assuming a transverse energy component $E_{0} \approx 0.2 \mathrm{eV}$. A value of $2 \mathrm{keV}$ was chosen as the beam energy to take advantage of the technique of brightness enhancement in reducing the beam diameter, without making the beam divergence unduly large. Canter et al [41] pointed out that the process of reducing the beam radius to the desired size by making use of the beam emittance invariance is limited by the aberrations that are encountered if the beam divergence is too large. A figure of $10^{\circ}$ has been established as an upper limit

$\S$ The PoIsson code was originally written by RF Holsinger of Lawrence Livermore National Laboratory and was subsequently modified by others at CERN. 
for the beam divergence before aberrations become prohibitively large. A beam energy of $2 \mathrm{keV}$ and a radius of $0.75 \mathrm{~mm}$ gives a divergence of $\sim 6^{\circ}$ for the BNL beam.

We scaled the tabulated Soa voltages appropriately [25] and, with the upstream edge of the magnetic field termination plate located $100 \mathrm{~mm}$ from the cathode plane, we determined the $(\rho, \alpha)$ values of the extreme phase space rays projected at the termination plate entrance. One such pair of rays for $2 \mathrm{keV}$ positrons is represented by the two full circles at the corners of the parallelogram on the idealized $(\rho, \alpha)$ phase space plot shown in figure 3. Also shown in the figure are open circles corresponding to a set of three non-extreme rays, which we used to test the bounding character of the extreme rays.

The results for the transport of the five phase space points of figure 3 appear in figure 4 , which illustrates the ray tracing output of the SLAC/Hermannsfeldt code as applied to the first $440 \mathrm{~mm}$ of the terminated magnetic field. The positron source moderator is assumed to be at the origin in figure 4 and the terminating plate is placed in the field-free region [25] a distance of $100 \mathrm{~mm}$ downstream. The ray tracing starts at a point $86 \mathrm{~mm}$ from the source moderator prior to entry into the terminated magnetic field. The procedure described by Canter [25] is used to determine the $(\rho, \alpha)$ starting values of the traces. For the purpose of analysis, it should be noted that the cusps that appear periodically for each ray are artefacts of the output format shown: namely radial against axial coordinate. The actual motion, of course, is helical. Close scrutiny of either of the focal

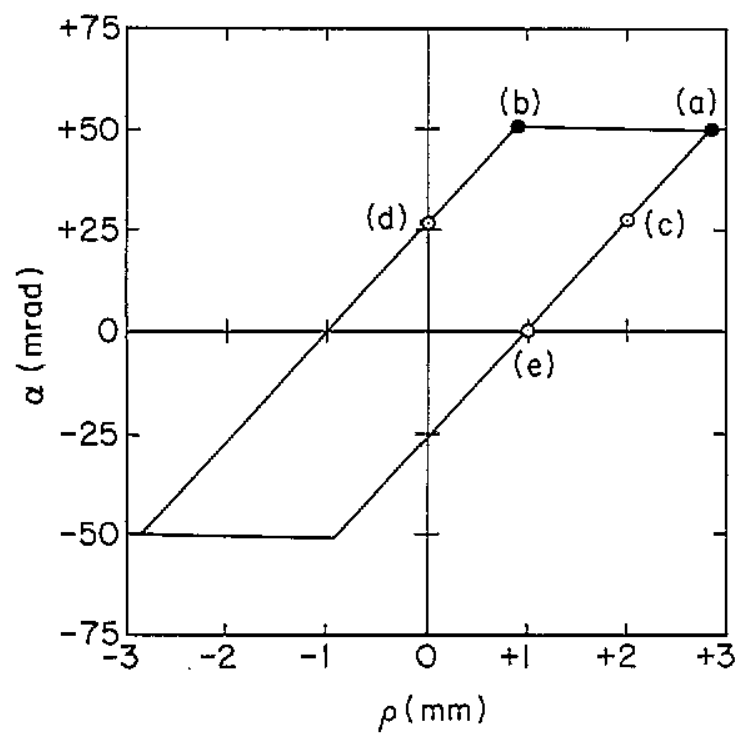

Figure 3. Idealized $(\rho, \alpha)$ phase space plot characterizing a $2 \mathrm{keV}\left(V_{\mathrm{c}}=2000 \mathrm{~V}\right)$ positron beam at the upstream edge of the entrance termination plate. The full circles represent the two extreme rays, while the open circles show a sample of three intermedlate rays used in testing the calculations of Canter et al [41]. The letters on the circles correspond to the propagated trajectories shown in figure 4 . For the plot shown, the modified Soa gun was tuned for optimal operation at an axial magnetic field of $50 \mathrm{G}$ as indicated on the right-hand vertical scale.

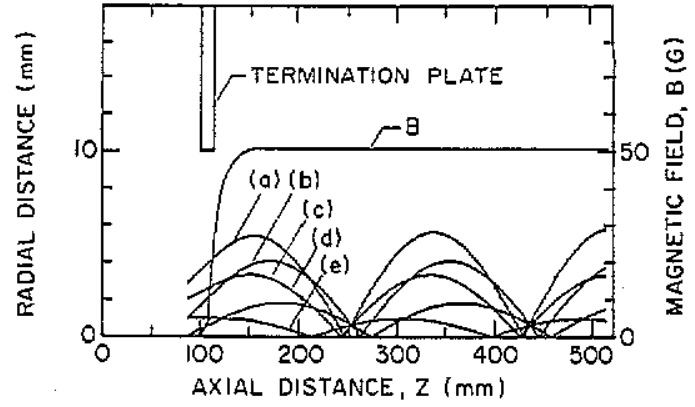

Figure 4. SLAC/Hermannsfeldt [39] trajectory calculations for $2 \mathrm{keV}\left(V_{\mathrm{c}}=2000 \mathrm{~V}\right)$ positrons entering the terminated magnetic field with an axial value of $50 \mathrm{G}$. The letters on the circles correspond to the $(\rho, \alpha)$ phase space designations at the upstream edge of the entrance termination plate, as given in Figure 3. The axial coordinate of the modified Soa gun cathode is taken to be $z=0$. At the focal planes $(z=250 \mathrm{~mm}$ and $z=435 \mathrm{~mm})$, close inspection shows that the extreme rays (a) and (b) bound the three remaining intermediate rays.

planes $(z=250 \mathrm{~mm}$, for example) shown in figure 4 reveals that the extreme phase space points of figure 3 do indeed bound the problem.

We carried out a detailed analysis by varying both the transport energy of the positrons and the strength of the magnetic field for different tunes of the modified Soa gun [4I]. Gun tunings that resulted in large opening angles for the bounding rays could only be used with magnetic fields less than $30 \mathrm{G}$; otherwise reflection of the positrons occurred at the entrance to the magnetic field. We wanted to have the option of using a relatively high magnetic field to provide the possibility of using a curved solenoidal transport system. (The drift velocity of the cyclotron guiding centre from the flux lines in a curved solenoidal field is inversely proportional to the strength of the magnetic field [6]). Gun tunings that resulted in the smallest opening angles of the bounding rays allowed us to use magnetic fields as large as $200 \mathrm{G}$ without reflection of the positrons on entry to the magnetic field. The beam radius at the remoderator on exit from the magnetic field, however, was in excess of the $0.75 \mathrm{~mm}$ limit, established in section 2, for these gun tunings. An optimum gun tune with intermediate opening angles was determined, in which it was possible to use magnetic fields up to $100 \mathrm{G}$ without reflection of the positrons at the entry to the magnetic field.

It is well known that the axial distance travelled by a charged particle in a uniform magnetic field, often referred to as the pitch distance, is inversely proportional to the field strength. When the pitch distance of the positrons became comparable with the termination distance of the magnetic field, we encountered difficulty in extracting the bounding rays from the magnetic field. An intermediate value of $50 \mathrm{G}$ was chosen for the magnetic field which satisfied all the criteria of the beam. In this context, we note that a $2 \mathrm{keV}$ beam inside a $50 \mathrm{G}$ guide field is consistent with the requirements of the 'guiding centre approximation' [19] and the preservation of the relative phase of cyclotron orbits [6] in a curved 
axial magnetic field, provided a mean radius of curvature of at least $5 \mathrm{~m}$ is employed.

Figure $5(a)$ illustrates the passage of the two bounding rays through the terminated magnetic field for a transport energy of $2 \mathrm{keV}$, a magnetic field strength of $50 \mathrm{G}$, and a remoderator potential $V_{\mathrm{r}}=0 \mathrm{~V}$. The two transition regions between the magnetic and electrostatic fields are the least well understood parts of the transport-we have therefore concentrated most of our attention on these two sections. We determined conditions which would ensure that the positrons could be successfully launched into the magnetic field and, following transport by the magnetic field, could be extracted onto a thin film transmission moderator in a region free of magnetic fields. We have not attempted to follow the paths of the positrons in the magnetic field, as we have assumed that the positrons follow the usual helical paths in an axial magnetic field. Figure $5(b)$ shows the corresponding paths for a transport energy of $3 \mathrm{keV}$, a magnetic field of $50 \mathrm{G}$, and a remoderator potential $V_{\mathrm{r}}=$ $+1 \mathrm{kV}$. In both figures the periodic behaviour of the beam inside the magnetic field is evident.

For the $2 \mathrm{keV}, 50 \mathrm{G}$ situation shown in Figure $5(a)$, we found the optimum performance to occur with the upstream edge of the exit termination plate positioned $170 \mathrm{~mm}$ downstream from the last focal plane inside the magnetic field. Then with the remoderator biased at $0 \mathrm{~V}$ and positjoned $14 \mathrm{~mm}$ away from the downstream edge of the exit termination plate we obtained a spot-size radius of less than $0.75 \mathrm{~mm}$ on the moderator, which is consistent with our design requirement. Moreover, as we varied the potential on the remoderator $V_{\mathrm{r}}$ between 0 and $+500 \mathrm{~V}$, the condition necessary for achieving the required dynamic energy range for the remoderated beam (assuming a crossed-beams interaction region at ground potential), we found that the spot-size radius varied by less than $5 \%$. Thus for $2 \mathrm{keV}$ transport, we found a design approach that meets the needs we established at the outset.

For the $3 \mathrm{keV}$ transport case illustrated in Figure $5(b)$ we found the optimum performance to occur with the upstream edge of the exit termination plate positioned $200 \mathrm{~mm}$ downstream from the last focal plane inside the magnetic field. We observed the smallest spot size to be slightly more than $1 \mathrm{~mm}$ in radius when the remoderator was biased at $+1000 \mathrm{~V}$ and positioned $18 \mathrm{~mm}$ away from the downstream edge of the exit termination plate.

\section{Conclusion}

We have shown how a hybrid EBE positron transport system was designed in conjunction with brightness enhancement remoderation, for use in crossed-beams low-energy positron-atom scattering experiments. The EBE configuration is essentially a solution to the well known problem of extracting a beam of positrons from a magnetic field. Novel schemes for producing more intense positron beams continue to encounter this problem [30]. Some of the principles we have examined,

(a)

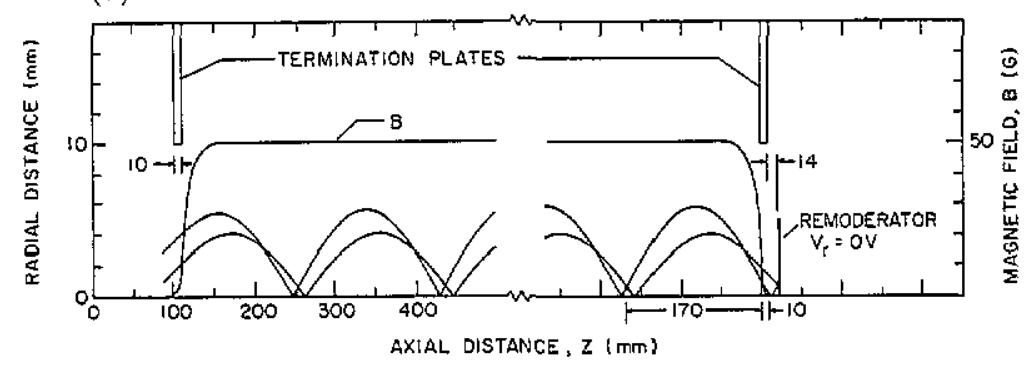

(b)

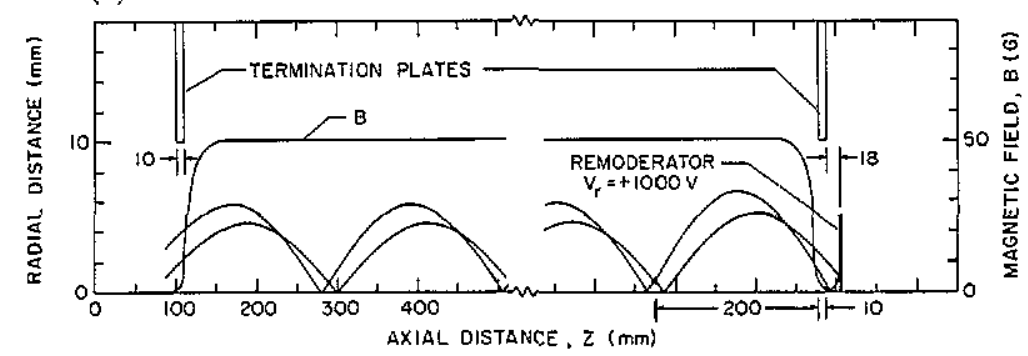

Figure 5. Magnetic transport trajectories calculated by the SLAC/Hermannsfeldt [39] for the two modified Soa gun extreme rays that correspond to optimal tuning conditions appropriate to a $50 \mathrm{G}$ internal axial magnetic field and beam energies of $2 \mathrm{keV}\left(V_{c}=2000 \mathrm{~V}\right)$ in (a) and $3 \mathrm{keV}\left(V_{c}=3000 \mathrm{~V}\right)$ in (b). In both (a) and (b) the axial coordinate is taken to be $z=0$ with the coordinates of all other elements (focal planes, termination plates, and remoderators) as shown. Note that in (a) the remoderator has a bias voltage $V_{r}=0 \mathrm{~V}$ while in $(b)$ it has a bias voltage $V_{r}=1000 \mathrm{~V}$. 
particularly those dealing with the magnetic field termination problem and the basic concept of the hybrid approach, have clear application in this regard.

\section{Acknowledgments}

This work was supported by the US National Science Foundation (Grant PHY-8603166), the City University of New York (Grant PSC-CUNY 6-67353) and the Irish National Board for Science and Technology. We wish to express our appreciation to Dr K G Lynn of Brookhaven National Laboratory for the hospitality he extended during our stay. We also wish to acknowledge helpful discussions with Dr Lynn as well as with Professor K F Canter of Brandeis University and Professor L O Roellig of the City College of CUNY.

\section{References}

[1] Canter K F, Coleman P G, Griffeth T C and Heyland G R 1972 Measurement of total cross sections for low energy positron-helium collisions J. Phys. B: At. Mol, Phys. 5 Li67-9

[2] Costello D G, Groce D E, Herring D F and McGowan J W 1972 Evidence for the negative work function associated with positrons in gold Phys. Rev. B 5 1433-9

[3] Pendyala S, Zitzewitz P W, McGowan J W and Orth P H R 1973 Low energy positrons from metallic moderators in a back scattering mode Phys. Lett. 43A 298-300

[4] Stein T S, Kauppila W E and Roellig L O 1974 Production of a monochromatic low energy positron beam using the $\mathrm{B}^{11}(\mathrm{p}, \mathrm{n}) \mathrm{C}^{11}$ reaction $R e v$. Sci. Instrum. 45 951-3

[5] Canter K F, Mills A P Jr and Berko S 1974 Efficient positronium formation by slow positrons incident on solid targets Phys. Rev. Lett. 33 7-10

[6] Canter K F and Mills A P Jr 1982 Slow positron beam design notes Can. J. Phys. 60 551-7

[7] Howell R H, Alvarez R A, Woodle K A, Dhawan S and Egan P O 1984 Intense positron beams: linacs Positron Scattering in Gases ed J W Humberston and M R C McDowell (New York: Plenum) pp 155-63

[8] Charlton M 1985 Experimental studies of positrons scattering in gases Rep. Prog. Phys. 48 737-93

[9] Mills A P Jr and Gullikson E M 1986 Solid neon moderator for producing slow positrons Appl. Phys. Lett. $491121-3$

[10] Canter K F, Brandes G R, Horsky T N, Lippel P H and Mills A P Jr 1987 The high brightness beam at Brandeis Atomic Physics with Positrons ed $\mathbf{J} \mathbf{W}$ Humberston and E A G Armour (New York: Plenum) pp 153-60

[11] Lynn K G, Weber M, Roellig L O, Mills A P Jr and Moodenbaugh A R 1987 A high intensity positron beam at the Brookhaven reactor Atomic Physics with Positrons ed J W Humberston and E A G Armour (New York: Plenum) pp 161-74

[12] Beling C D, Simpson R I, Charlton M, Jacobsen F M and Griffith T C 1987 A field assisted moderator for low-energy positron beams Appl. Phys. A $42111-6$
[13] Schultz P J and Lynn K G 1988 Interaction of positron beams with surfaces, thin films, and interfaces Rev. Mod. Phys. $6070 \mathrm{I}-79$

[14] Charlton M and Laricchia G 1990 Positron impact phenomena J. Phys, B: At. Mol. Opt. Phys. 23 1045-78

[15] Gramsch E, Lynn K G, Throwe J and Kanazawa I 1991 Positron diffusion in solid and liquid $\mathrm{Ga}$ and Bi Phys. Rev. Lett. 67 1282-5

[16] Merrison J P, Chariton M and Laricchia G 1991 A new timed slow positron beam Meas. Sci. Technol. 2 175-7

[17] Raith W 1987 Positron-impact ionization and positronium formation Atomic Physics with Positrons ed J W Humberston and E A G Armour (New York: Plenum) pp 1-14

[18] Sinapius G, Spicher G and Ravn H L 1986 Positron sources for atomic physics experiments $J$. Phys. E: Sci. Instrum. 19 987-8

[19] Kauppila W E and Stein T S 1987 Positron-atom differential scattering measurements Atomic Physics with Positrons ed J W Humberston and E A G Armour (New York: Plenum) pp 27-39

[20] Charlton M, Laricchia G, Zafar N and Jacobsen F M 1987 Inelastic positron collisions in gases Atomic Physics with Positrons ed J W Humberston and E A G Armour (New York: Plenum) pp 15-25

[21] Mills A P Jr 1979 Efficient generation of low-energy positrons Appl. Phys. Lett. 35 427-9

[22] Mills A P Jr 1980 Further improvements in the efficient production of low-energy positron moderators Appl. Phys. Lett. 37 667-8

[23] Kauppila W E, Steín T S, Jession G, Dababneh M S and Pol V 1977 Transmission experiment for measuring total positron-atom collision cross sections in a curved, axial magnetic field Rev. Sci. Instrum. 48 822-8

[24] Lyan K G and Lutz H 1980 Slow-positron apparatus for surface studies Rev. Sci. Instrum. 51 977-82

[25] Canter K F 1986 Slow positron optics Positron Studies of Solids, Surfaces and Atoms ed A P Mills Jr et al (Singapore: World Scientific) pp 102-20

[26] Coleman P G, McNutt J D, Hutton J T, Díana L M and Fry J L 1980 A time-of-flight spectrometer for the measurement of angular distributions of scattered slow positrons and electrons Rev. Sci. Instrum. 51 935-43

[27] Sueoka O 1987 Total and inelastic positron scattering cross sections Atomic Physics with Positrons ed J W Humberston and E A G Armour (New York: Plenum) pp 41-54

[28] Hyder G M A, Dababneh M S, Hsieh Y-F, Kauppila W E, Kwan C K, Mahdavi-Hezaveh $M$ and Stein T S 1986 Positron differential elastic-scattering cross section measurements for argon Phys. Rev. Lett. 57 2252-5

[29] Schwab A, Höner P, Raith W and Sinapius G 1987 Elastic differential positron scattering from argon Atomic Physics with Positrons ed J W Humberston and $E A G$ Armour (New York: Plenum) pp 429-31

[30] Taqqu D 1990 High efficiency positron moderation Helv. Phys. Acta 60 442-7

[31] Hughes V W, Long R L Jr, Lubell M S, Posner M and Raith W 1972 Polarized electrons from photoionization of polarized alkali atoms Phys. Rev. A 5 195-222

[32] Sinapius G 1984 Applications of intense positron beams in atomic physics experiments Positron Scattering in Gases ed J W Humberston and M R C McDowell (New York: Plenum) pp 211-8 
[33] Mills A P Jr 1980 Brightness enhancement of slow positron beams Appl. Phys. 23 189-91

[34] Lynn K G and Wachs A 1982 Positron remission brightness enhancement method Appl. Phys. A 29 93-8

[35] Chen D M, Lynn K G, Pareja R and Nielsen B 1985 Measurement of positron re-emission from thin single-crystal W(100) films Phys. Rev. B 31 4123-30

[36] Frieze W E, Gidley D W and Lynn K G 1985 Positronbeam-brightness enhancement: Low-energy positron diffraction and other applications Phys. Rev. B 31 5628-33

[37] Canter K F, Horsky T, Lippel P H, Crane W S and Mills A P Jr 1986 Development of high brightness slow positron beams Proc. Third Int. Workshop on
Positron (Electron)-Gas Scattering ed W E Kauppila et al (Singapore: World Scientific) pp 202-11

[38] Lynn K G, Nielsen B and Quateman J H 1985 Development and use of a thin-film transmission positron moderator Appl. Phys. Lett. 47 239-40

[39] Hermannsfeldt W B 1979 SLAC Report 226 (Stanford Linear Accelerator Center, Stanford, CA)

[40] Klemperer O and Barnett M E 1971 Electron Optics 3rd edn (Cambridge: Cambridge University Press) pp $110 \mathrm{ff}$

[41] Canter K F, Lippel P H, Crane W S, and Mills A P Jr 1986 Modified Soa immersion lens positron gun Positron Studies of Solids, Surfaces and Atoms ed A P Mills Jr et al (Singapore: World Scientific) pp 199-206 